\title{
Equivalence of the Local Markov Inequality and a Kolmogorov Type Inequality in the Complex Plane
}

\author{
Leokadia Białas-Cież • Raimondo Eggink
}

Received: 2 August 2011 / Accepted: 25 January 2012 / Published online: 10 May 2012

(C) The Author(s) 2012. This article is published with open access at SpringerLink.com

\begin{abstract}
We prove that a compact subset of the complex plane satisfies a local Markov inequality if and only if it satisfies a Kolmogorov type inequality. This result generalizes a theorem established by Bos and Milman in the real case. We also show that every set satisfying the local Markov inequality is a sum of Cantor type sets which are regular in the sense of the potential theory.
\end{abstract}

Keywords Markov inequality $\cdot$ Kolmogorov inequality • Green function • L-regularity of sets $\cdot$ Holomorphic functions $\cdot$ Cantor sets

Mathematics Subject Classifications (2010) $41 \mathrm{~A} 17 \cdot 31 \mathrm{C} 15 \cdot 30 \mathrm{E} 10$

\section{Introduction}

Let $K \subset \mathbb{C}^{N}$ be a compact set. The pluricomplex Green function (with pole at infinity) of $K$ can be defined by

$$
V_{K}(z):=\sup \{u(z): u \in \mathcal{L} \text { and } u \leq 0 \text { on } K\}, \quad z \in \mathbb{C}^{N},
$$

where $\mathcal{L}$ is the family of all plurisubharmonic functions in $\mathbb{C}^{N}$ of logarithmic growth at the infinity, i.e.

$$
\mathcal{L}:=\left\{u \text { plurisubharmonic in } \mathbb{C}^{\mathrm{N}}: \mathrm{u}(\mathrm{z})-\log \|\mathrm{z}\| \leq \mathcal{O}(1) \text { as }\|\mathrm{z}\| \rightarrow \infty\right\} .
$$

In the one dimensional case $V_{K}$ coincides with the Green function $g_{K}$ of the unbounded component of $\hat{\mathbb{C}} \backslash K$ with logarithmic pole at infinity (as usual $\hat{\mathbb{C}}=\mathbb{C} \cup\{\infty\}$ is the Riemann sphere).

L. Białas-Cież $(\varangle) \cdot$ R. Eggink

Institute of Mathematics, Faculty of Mathematics and Computer Science, Jagiellonian

University, ul. Łojasiewicza 6, 30-348 Kraków, Poland

e-mail: leokadia.bialas-ciez@im.uj.edu.pl 
The (pluricomplex) Green function is closely related to polynomials in view of the formulas

$$
\begin{gathered}
V_{K}(z)=\lim _{n \rightarrow \infty} \frac{1}{n} \log \Phi_{n}(K, z), \\
\Phi_{n}(K, z)=\sup \left\{\frac{|P(z)|}{\|P\|_{K}}: P: \mathbb{C}^{N} \rightarrow \mathbb{C} \text { polynomial of degree } n,\left.P\right|_{K} \neq 0\right\}
\end{gathered}
$$

and $\|\cdot\|_{K}$ is the maximum norm on $K$ (see [16] or [13, Theorem 5.1.7]).

A topic of great interest is the relationship between the regularity of the (pluricomplex) Green function and some Markov-type polynomial inequalities. These inequalities can be defined not only on subsets of $\mathbb{C}^{N}$ but also of $\mathbb{R}^{N}$ and in order to compare them with some properties of $V_{K}$, we consider $\mathbb{R}^{N}$ as a subspace of $\mathbb{C}^{N}$ in the usual way, i.e. $\mathbb{R}^{N}=(\mathcal{R} e \mathbb{C})^{N} \subset \mathbb{C}^{N}$, where $\operatorname{Re} z$ is the real part of $z$.

A compact set $E \subset \mathbb{K}^{N}$ ( $\mathbb{K}=\mathbb{R}$ or $\mathbb{C}$ ) is said to have the Global Markov Property (GMP) if for every polynomial $P$ of $N$ variables

$$
\||\nabla P|\|_{E} \leq M(\operatorname{deg} P)^{s}\|P\|_{E},
$$

where $\nabla P:=\left(\frac{\partial P}{\partial z_{1}}, \ldots, \frac{\partial P}{\partial z_{N}}\right),|\nabla P|=\left(\sum_{j=1}^{N}\left|\frac{\partial P}{\partial z_{j}}\right|^{2}\right)^{1 / 2}$ and the positive constants $M, s$ are independent of $P$.

Sets with this property play an important role in the constructive theory of functions, especially in problems of polynomial approximation and extension of $\mathcal{C}^{\infty}$ functions (see e.g. $[1,6,8,14,21]$ ). The GMP has found some important applications also in numerical analysis because of its connection with polynomial interpolation and approximation of $\mathcal{C}^{\infty}$ and holomorphic functions (e.g. [5, 9]).

It is worth noticing that by a result of Pleśniak [14], the global Markov property can be rewritten in the following form

$$
\sup \left\{\Phi_{n}(E, z): \operatorname{dist}(z, E) \leq \frac{1}{n^{s}}\right\} \leq M,
$$

where $M, s>0$ are independent of $n$.

The last inequality and formula (1) suggest that the GMP of $K$ may be closely related to the regularity of $V_{K}$. Indeed, the Hölder continuity of $V_{K}$ implies the global Markov property of $K$ (see [17, Lemma 3]) and every set with the GMP seems to be L-regular but that has been proved so far only for compact subsets of $\mathbb{R}$ (see [3]) (we say that $K$ is L-regular if $V_{K}$ is continuous). In the general (complex) case the question about the L-regularity of sets with the global Markov property remains an open problem posed explicitly in [14] and [19]. However, every compact set $K \subset \mathbb{C}$ with the GMP is not polar [2], which is a necessary condition for the continuity of $g_{K}$ (see e.g. [15, Theorems 4.4.2,3]).

On the other hand, it turns out that every compact subset of $\mathbb{C}$ with a local version of the Markov property (LMP, see Definition 1.4) is L-regular (see [3] or Section 4 in this paper). The implication LMP $\Rightarrow$ GMP is immediate but any attempt to prove the converse poses considerable difficulties.

Bos and Milman [7, 8] Bos and Milman (The equivalence of Markov and Sobolev type inequalities on compact sets in Rn (preprint)), Bos and Milman (A Geometric Interpretation and the Equality of Exponents in Markov and Gagliardo-Nirenberg (Sobolev) Type Inequalities for Singular Compact Domains (preprint)) have proved 
that a local Markov inequality (Definition 1.1) is equivalent to the GMP in the real case. The proof is hard and requires demonstration of the equivalence of the local Markov inequality, a Kolmogorov type inequality (Definition 1.2) and an extension property for $\mathcal{C}^{\infty}(K)$ functions. We were intrigued to obtain a corresponding result for sets in the complex plane because of the intricate interconnectedness of multiple distinct global and local properties: Markov inequalities, Kolmogorov type inequalities, polynomial approximation, extension operators, geometric properties and, ultimately, the behavior of the Green function, i.e. L-regularity, Hölder continuity and the Łojasiewicz-Siciak property. However, a simple adaptation to the complex case of the proof given by Bos and Milman does not seem possible. In this paper we show that the LMP is equivalent to a Kolmogorov type property for any compact subset of $\mathbb{C}$. This is the first step to show the equivalence (under an additional but necessary assumption) of the LMP and the GMP in the complex plane and, in consequence, to prove the L-regularity of sets with the global Markov property in $\mathbb{C}$. The next steps of the demonstration of this equivalence will be the subject of two papers that are now in their final stage of preparation (see Bialas-Ciez and Eggink (Łojasiewicz-Siciak inequality of Green's function and a version of Jackson's theorem in the complex plane (Preprint)), Bialas-Ciez and Eggink (Equivalence of local and global Markov inequality in the complex plane (Preprint))).

We first recall the definitions of the local Markov inequality and the Kolmogorov type inequality given by Bos and Milman.

We denote by $\mathcal{P}_{n}\left(\mathbb{R}^{N}\right)$ the space of all polynomials of $N$ real variables with real coefficients of degree at most $n$. Let $\mathbb{N}_{0}:=\{0,1,2, \ldots\}$. For $\alpha=\left(\alpha_{1}, \ldots, \alpha_{N}\right) \in \mathbb{N}_{0}^{N}$

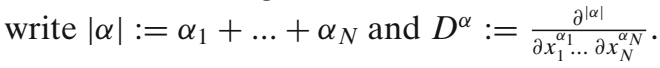

Definition 1.1 ([Bos and Milman (A Geometric Interpretation and the Equality of Exponents in Markov and Gagliardo-Nirenberg (Sobolev) Type Inequalities for Singular Compact Domains (preprint)) Definition 2.3] or Bos and Milman (The equivalence of Markov and Sobolev type inequalities on compact sets in Rn (preprint)) [7]) Let $m \geq 1$. A compact set $K \subset \mathbb{R}^{N}$ admits the Local Markov Inequality $(\operatorname{LMI}(m)$ in short) if there exist $c_{1}, \kappa \geq 1$ such that for every $n \in \mathbb{N}, x_{0} \in K, r \in(0,1], P \in \mathcal{P}_{n}\left(\mathbb{R}^{N}\right)$ and $\alpha \in \mathbb{N}_{0}^{N}$

$$
\left|D^{\alpha} P\left(x_{0}\right)\right| \leq\left(\frac{c_{1} n^{\kappa}}{r^{m}}\right)^{|\alpha|}\|P\|_{K \cap B\left(x_{0}, r\right)},
$$

where $B\left(x_{0}, r\right):=\left\{x:\left|x-x_{0}\right| \leq r\right\}$ and $\|g\|_{F}=\sup \{|g(x)|: x \in F\}$.

Note that here and below we do not assume $m \geq 1$ to be an integer.

For $f \in \mathcal{C}^{\infty}\left(\mathbb{R}^{N}\right), j \in \mathbb{N}$ we put

$$
|f|_{K, j}:=\sum_{|\alpha|=j}\left\|D^{\alpha} f\right\|_{K}, \quad\|f\|_{K, j}:=\|f\|_{K}+|f|_{K, j}, \quad\|f\|_{K, 0}:=\|f\|_{K} .
$$

Consider also the quotient norms

$$
\|f\|_{K, j}:=\inf \left\{\|g\|_{\operatorname{conv}(K), j}: g \in \mathcal{C}^{\infty}\left(\mathbb{R}^{N}\right), \quad g /{ }_{K} \equiv f /{ }_{K}\right\}, \quad j \in \mathbb{N}_{0},
$$

where $\operatorname{conv}(K)$ is the convex hull of $K$. 
Definition 1.2 ([Bos and Milman (A Geometric Interpretation and the Equality of Exponents in Markov and Gagliardo-Nirenberg (Sobolev) Type Inequalities for Singular Compact Domains (preprint)), Definition 2.15] or Bos and Milman (The equivalence of Markov and Sobolev type inequalities on compact sets in $\mathrm{Rn}$ (preprint)) [7]) A compact set $K \subset \mathbb{R}^{N}$ admits the Kolmogorov Inequality in Quotient Norms $(\mathrm{KIQ}(m), m \geq 1)$ if there are constants $c_{1}, \kappa \geq 1$ such that for all $f \in \mathcal{C}^{\infty}\left(\mathbb{R}^{N}\right)$

$$
|f|_{K, j} \leq\left(c_{1} l^{\kappa}\right)^{j}\|f\|_{K}^{1-\frac{m j}{l}}\|f\|_{K, l}^{\frac{m j}{l}} \quad \text { for all } j, l \in \mathbb{N} \text { such that } m j \leq l .
$$

This property has been called by Bos and Milman a Sobolev inequality in the quotient topology. However, M. Baran noted that condition (3) is more closely related to Kolmogorov (or Landau) type inequalities.

Theorem 1.3 ([Bos and Milman (A Geometric Interpretation and the Equality of Exponents in Markov and Gagliardo-Nirenberg (Sobolev) Type Inequalities for Singular Compact Domains (preprint)), Theorem A] or [7, Theorem 3.9]) If $K$ is a compact subset of $\mathbb{R}^{N}$ and $m \geq 1$, then

(a) $K I Q(m) \Rightarrow \operatorname{LMI}\left(m^{\prime}\right)$ for any $m^{\prime}>m$,

$K I Q(1) \Rightarrow L M I(1)$

(b) $\operatorname{LMI}(m) \Rightarrow K I Q(m)$.

Our first attempts to prove an analogous theorem in the complex plane failed owing to several obstacles. The difficulty was that we did not know how to replace the space of $\mathcal{C}^{\infty}$ functions by another class of functions that would be well adapted to the Kolmogorov inequality in view of a proof of the local Markov inequality. An idea to use the functions of class $\mathcal{A}^{\infty}$ appeared first in [11]. Another obstacle was the fact that a cutoff function multiplied by a complex polynomial may not be of class $\mathcal{A}^{\infty}$ on a fixed compact set. We get over this problem by a sequence of lemmas.

Let $E$ be a compact set in the complex plane. From now on, we will use the following notation.

For $\alpha=\left(\alpha_{1}, \alpha_{2}\right) \in \mathbb{N}_{0}^{2}, f \in \mathcal{C}^{\infty}(\mathbb{C})$ let $z^{\alpha}:=z^{\alpha_{1}} \bar{z}^{\alpha_{2}}$ and $D^{\alpha} f:=\frac{\partial^{|\alpha|} f}{\partial z^{\alpha_{1}} \partial \bar{z}^{\alpha_{2}}}$. As usual, $\left(\begin{array}{l}\alpha \\ \beta\end{array}\right)=\left(\begin{array}{c}\alpha_{1} \\ \beta_{1}\end{array}\right)\left(\begin{array}{l}\alpha_{2} \\ \beta_{2}\end{array}\right)$ and $\beta \leq \alpha$ iff $\beta_{1} \leq \alpha_{1}$ and $\beta_{2} \leq \alpha_{2}$ for $\alpha=\left(\alpha_{1}, \alpha_{2}\right) \in \mathbb{N}_{0}^{2}, \beta=\left(\beta_{1}, \beta_{2}\right) \in$ $\mathbb{N}_{0}^{2}$. Of course if $f$ is a holomorphic function at $z_{0}$ then $D^{\alpha} f\left(z_{0}\right)=0$ whenever $\alpha_{2}>0$. Denote by $\mathcal{P}_{n}(\mathbb{C})$ the space of all holomorphic polynomials with complex coefficients of degree at most $n$.

Definition 1.4 Let $m, \kappa \geq 1$. A compact set $E \subset \mathbb{C}$ is said to admit the Local Markov Property $\operatorname{LMP}(m, \kappa)$, if for all $n \in \mathbb{N}, z_{0} \in E, r \in(0,1], P \in \mathcal{P}_{n}(\mathbb{C})$ and $j \in \mathbb{N}$

$$
\left|P^{(j)}\left(z_{0}\right)\right| \leq\left(\frac{c_{1} n^{\kappa}}{r^{m}}\right)^{j}\|P\|_{E \cap B\left(z_{0}, r\right)}
$$

with $c_{1}>0$ depending only on $E$. We will write that the set $E$ admits $\operatorname{LMP}(m)$ if it admits $\operatorname{LMP}(m, \kappa)$ for some $\kappa \geq 1$.

Evidently, if $E \subset \mathbb{R}$ then $\operatorname{LMP}(m)$ is equivalent to $\operatorname{LMI}(m)$ with $N=1$. 
A smooth function $f$ is flat at $z_{0} \in \mathbb{C}$ if $D^{\alpha} f\left(z_{0}\right)=0$ for all $\alpha \in \mathbb{N}_{0}^{2}$. We call $f \in$ $\mathcal{C}^{\infty}(\mathbb{C}) \bar{\partial}$-flat on $E$ if $\frac{\partial f}{\partial \bar{z}}$ is flat at every point of $E$. We will denote by $\mathcal{A}^{\infty}(E)$ the set of all smooth functions $\bar{\partial}$-flat on $E$

$$
\mathcal{A}^{\infty}(E):=\left\{f \in \mathcal{C}^{\infty}(\mathbb{C}): f \text { is } \bar{\partial} \text {-flat on } E\right\}
$$

(compare with [21, Section 5] and [18]). For $f \in \mathcal{C}^{\infty}(\mathbb{C})$ and $j \in \mathbb{N}$ let

$$
\begin{aligned}
& |f|_{E, j}:=\sum_{\alpha \in \mathbb{N}_{0}^{2},|\alpha|=j}\left\|D^{\alpha} f\right\|_{E}, \quad|f|_{E, 0}:=\|f\|_{E} \\
& \|f\|_{E, j}:=\|f\|_{E}+|f|_{E, j}, \quad\|f\|_{E, 0}:=\|f\|_{E}
\end{aligned}
$$

and for $f \in \mathcal{A}^{\infty}(E)$ let

$$
\|f\|_{E, j}:=\inf \left\{\|g\|_{\operatorname{conv}(E), j}: g \in \mathcal{A}^{\infty}(E), \quad g /{ }_{E} \equiv f /{ }_{E}\right\}, \quad\|f\|_{E, 0}:=\|f\|_{E} .
$$

Definition 1.5 A compact set $E \subset \mathbb{C}$ admits the Kolmogorov Property in Quotient Norms $\operatorname{KPQ}(m, \kappa)$, where $m, \kappa \geq 1$, if there exists a constant $c_{1} \geq 1$ such that for all $f \in \mathcal{A}^{\infty}(E)$

$$
|f|_{E, j} \leq\left(c_{1} l^{\kappa}\right)^{j}\|f\|_{E}^{1-\frac{m j}{l}}\|f\|_{E, l}^{\frac{m j}{l}} \quad \text { for all } j, l \in \mathbb{N} \text { such that } m j \leq l .
$$

We will write that the set $E$ admits $\operatorname{KPQ}(m)$ if it admits $\operatorname{KPQ}(m, \kappa)$ for some $\kappa \geq 1$.

It is worth noticing that for $E \subset \mathbb{R}$ we have $\mathcal{A}^{\infty}(E) /_{\mathbb{R}}=\mathcal{C}^{\infty}(\mathbb{R})$ (see [18]) and the $\operatorname{KPQ}(m)$ is equivalent to the $\operatorname{KIQ}(m)$ with $N=1$.

We are now ready to state an analogue of Theorem 1.3.

Theorem 1.6 If $E$ is a compact set in $\mathbb{C}$ and $m \geq 1$, then

(a) $K P Q(m) \Rightarrow \operatorname{LMP}\left(m^{\prime}\right)$ for any $m^{\prime}>m$, $K P Q(1) \Rightarrow L M P(1)$

(b) $L M P(m) \Rightarrow K P Q(m)$.

This theorem is an easy consequence of a more general result. In order to formulate it, we need some additional definitions of Kolmogorov type inequalities. These properties will be necessary in the next stages of the proof of the equivalence of the local and global Markov inequalities (see Bialas-Ciez and Eggink (LojasiewiczSiciak inequality of Green's function and a version of Jackson's theorem in the complex plane (Preprint)), Białas-Ciez and Eggink (Equivalence of local and global Markov inequality in the complex plane (Preprint))).

Let $F \subset \mathbb{C}$ be a compact set. Denote by $\mathcal{H}^{\infty}(F)$ the family of smooth functions that are holomorphic in some open neighbourhood of the set $F$, i.e.

$$
\mathcal{H}^{\infty}(F):=\left\{f \in \mathcal{C}^{\infty}(\mathbb{C}): \frac{\partial f}{\partial \bar{z}} \equiv 0 \text { in some open neighbourhood of } F\right\} .
$$

Of course $\mathcal{H}^{\infty}(F) \subset \mathcal{A}^{\infty}(F)$. For $f \in \mathcal{H}^{\infty}(F)$ and $j \in \mathbb{N}$ let

$$
\left\langle\langle f\rangle_{F, j}:=\inf \left\{\|g\|_{\operatorname{conv}(F), j}: g \in \mathcal{H}^{\infty}(F), g /_{F} \equiv f /{ }_{F}\right\},\langle\langle f\rangle\rangle_{F, 0}:=\|f\|_{F} .\right.
$$


Clearly, if $f \in \mathcal{H}^{\infty}(F)$ we have $\|f\|_{F, j} \leq\left\langle\langle f\rangle_{F, j}\right.$. For $\delta \in(0,1]$ we define $F_{\delta}$ to be the set $\{z \in \mathbb{C}: \exists w \in F|z-w| \leq \delta\}$.

The next definition is a variant of the Kolmogorov Property in Quotient Norms KPQ adapted to holomorphic functions from $\mathcal{H}^{\infty}\left(E_{\delta}\right)$. We additionally require the constants to be dependent on $\delta$ in a specific way.

Definition 1.7 A compact set $E \subset \mathbb{C}$ admits the Kolmogorov Property in Quotient Norms for Holomorphic Functions $\operatorname{KPQH}(m, s, \kappa)$, where $m, \kappa \geq 1, s \geq 0$, if there exists a constant $c_{1} \geq 1$ such that for all $\delta \in(0,1], f \in \mathcal{H}^{\infty}\left(E_{\delta}\right)$

$$
|f|_{E, j} \leq\left(\frac{c_{1} l^{\kappa}}{\delta^{s}}\right)^{j}\|f\|_{E}^{1-\frac{m j}{l}}\langle\langle f\rangle\rangle_{E_{\delta}, l}^{\frac{m j}{l}} \quad \text { for all } j, l \in \mathbb{N} \text { such that } m j \leq l .
$$

We give also a weaker version of the KPQH. Namely, we replace the norms $\langle\langle\cdot\rangle\rangle_{E_{\delta}, l}$ by $\|\cdot\|_{E_{\delta}}$. We increase the first exponent by a factor $c_{o}$ only in order to have better control over the constants (see Białas-Ciez and Eggink (Equivalence of local and global Markov inequality in the complex plane (Preprint))).

Definition 1.8 A compact set $E \subset \mathbb{C}$ admits the Kolmogorov Property for Holomorphic Functions $\operatorname{KPH}(m, s, \kappa)$, where $m, s, \kappa \geq 1$, if there exist constants $c_{o} \geq 0, c_{1} \geq 1$ such that for all $\delta \in(0,1], f \in \mathcal{H}^{\infty}\left(E_{\delta}\right)$ and

$$
|f|_{E, j} \leq\left(\frac{c_{1} l^{\kappa}}{\delta^{s}}\right)^{j+c_{o}}\|f\|_{E}^{1-\frac{m j}{l}}\|f\|_{E_{\delta}}^{\frac{m j}{l}} \quad \text { for all } j, l \in \mathbb{N} \text { such that } m j \leq l .
$$

The statement given below is a refinement of Theorem 1.6 and expresses the equivalence not only of the LMP and the KPQ but also of the KPQH and KPH.

Theorem 1.9 If $E$ is a compact set in $\mathbb{C}$ and $m, \kappa \geq 1$, then

(a) $K P H(m, s, \kappa) \Rightarrow L M P\left(m^{\prime}, \kappa^{\prime}\right)$ for all $s \geq 1$ and $m^{\prime}>s, \kappa^{\prime}>\kappa+3 s$,

$K P H(m, 1, \kappa)$ with $c_{o}=0 \Rightarrow \operatorname{LMP}\left(1, \kappa^{\prime}\right)$ for any $\kappa^{\prime}>\kappa+3$,

(b) $\operatorname{LMP}(m, \kappa) \Rightarrow K P Q(m, \kappa)$,

(c) $K P Q(m, \kappa) \Rightarrow K P Q H(m, s, \kappa)$ for all $s \geq 0$,

(d) $K P Q H(m, s, \kappa) \Rightarrow K P H(m, m+s, \kappa+5 m)$ with $c_{o}=0$ for all $s \geq 0$.

In particular $\operatorname{KPQH}(1,0, \kappa) \Rightarrow \operatorname{KPH}(1,1, \kappa+5)$ with $c_{o}=0 \Rightarrow \operatorname{LMP}\left(1, \kappa^{\prime}\right) \Rightarrow$ $K P Q\left(1, \kappa^{\prime}\right) \Rightarrow K P Q H\left(1, s, \kappa^{\prime}\right)$ for all $s \geq 0$ and for any $\kappa^{\prime}>\kappa+8$.

In Sections 2, 3 and 4 we prove the statements a, b and $\mathrm{d}$ of Theorem 1.9 respectively. The Proof of Theorem 1.9.c is immediate because $\mathcal{H}^{\infty}\left(E_{\delta}\right) \subset \mathcal{H}^{\infty}(E) \subset$ $\mathcal{A}^{\infty}(E)$ and consequently for all $l \in \mathbb{N}, \delta \in(0,1]$ and $f \in \mathcal{H}^{\infty}\left(E_{\delta}\right)$ we have $\|f\|_{E, l} \leq$ $\left\langle\langle f\rangle_{E, l} \leq\left\langle\langle f\rangle_{E_{\delta}, l}\right.\right.$.

Section 5 is devoted to a geometric property of sets with the $\operatorname{LMP}(m)$. We can see at once that if $E \subset \mathbb{C}$ admits the $\operatorname{LMP}(m)$ then it is an $m$-perfect set, i.e. there exists $c>0$ such that for all $z_{0} \in E$ and $r \in(0,1]$

$$
\left\{z \in E: \frac{r^{m}}{c} \leq\left|z-z_{0}\right| \leq r\right\} \neq \emptyset .
$$


$\mathrm{m}$-Perfectness seems to be an interesting property of $E$ but it is not closely connected with the $\operatorname{LMP}(m)$ in view of L-regularity. Namely, the $\operatorname{LMP}(m)$ implies L-regularity (see [3, Theorem 2.2]) and there exist sets which are $m$-perfect for some $m$ without being L-regular (see [19]). In Section 5, we introduce the notion of $(m, \infty)$-perfectness, which is more closely related with the $\operatorname{LMP}(m)$ than $m$-perfectness. The Lregularity of $(m, \infty)$-perfect sets is a consequence of [3, Proposition 3.6]. In Section 5 we prove that all sets with the $\operatorname{LMP}(m)$ are $(m, \infty)$-perfect.

\section{The KPH Implies the LMP}

We start with the construction of Fekete extremal points which are a useful tool in proving Theorem 1.9.a. Let $n \in \mathbb{N}$ and $E \subset \mathbb{C}$ be a compact set containing at least $n+1$ points. Put

$$
V\left(x_{0}, x_{1}, \ldots, x_{n}\right):=\prod_{0 \leq \mu<v \leq n}\left(x_{v}-x_{\mu}\right)
$$

Points $z_{0}, z_{1}, \ldots, z_{n} \in E$ such that

$$
\left|V\left(z_{0}, z_{1}, \ldots, z_{n}\right)\right|=\max \left\{\left|V\left(x_{0}, x_{1}, \ldots, x_{n}\right)\right|: x_{0}, x_{1}, \ldots, x_{n} \in E\right\}
$$

are called Fekete extremal points of order $n$ for $E$. We denote by $L_{\mu}, \mu=0, \ldots, n$, the Lagrange polynomials associated to the Fekete extremal points $z_{0}, \ldots, z_{n}$, i.e.

$$
L_{\mu}(z):=\frac{V\left(z_{0}, \ldots, z_{\mu-1}, z, z_{\mu+1}, \ldots, z_{n}\right)}{V\left(z_{0}, \ldots, z_{n}\right)} \prod_{\nu=0, \nu \neq \mu}^{n} \frac{z-z_{\nu}}{z_{\mu}-z_{\nu}}
$$

It is easy to see that $\left\|L_{\mu}\right\|_{E}=1$ for every $\mu=0,1, \ldots, n$.

In order to prove the implication $\mathrm{KPH} \Rightarrow \mathrm{LMP}$, we need to do some preparations. The first proposition says that one can deduce a certain pointwise Markov inequality for the set $E \subset \mathbb{C}$ from a polynomial Markov-type estimate for a special circular projection of $E$.

Proposition 2.1 Let $E \subset \mathbb{C}$ be a compact set and $z_{0} \in E, r>0, n \in \mathbb{N}$ be fixed. Put

$$
T=T\left(z_{0}, r\right):=\left\{t \in[0, r]: \exists z \in E:\left|z-z_{0}\right|=t\right\} .
$$

Assume that $T$ contains at least $n+1$ different points and $t_{0}, \ldots, t_{n}$ are Fekete extremal points of order $n$ for $T, t_{0}=\min \left\{t_{0}, \ldots, t_{n}\right\}$. If there exists a constant $C_{n}>0$ such that for all Lagrange polynomials $L_{1}, \ldots, L_{n}$ associated to $t_{0}, \ldots, t_{n}$ we have

$$
\left|L_{\mu}^{\prime}(0)\right| \leq C_{n}\left\|L_{\mu}\right\|_{T}, \quad \text { as } \mu=1, \ldots, n .,
$$

then for every polynomial $P \in \mathcal{P}_{n}(\mathbb{C})$ it follows that

$$
\left|P^{\prime}\left(z_{0}\right)\right| \leq 2 n C_{n}\|P\|_{E \cap B\left(z_{0}, r\right)} .
$$


Proof We first observe that $t_{0}=0$. Choose $z_{1}, \ldots, z_{n} \in E \cap B\left(z_{0}, r\right)$ such that $\mid z_{v}-$ $z_{0} \mid=t_{v}$ for $v=1, \ldots, n$. Fix $P \in \mathcal{P}_{n}(\mathbb{C})$. It is easy to check that

$$
P^{\prime}\left(z_{0}\right)=\sum_{\mu=1}^{n}\left[\frac{P\left(z_{\mu}\right)-P\left(z_{0}\right)}{z_{\mu}-z_{0}} \prod_{\nu=1, \nu \neq \mu}^{n} \frac{z_{0}-z_{\nu}}{z_{\mu}-z_{\nu}}\right] .
$$

Hence

$$
\left|P^{\prime}\left(z_{0}\right)\right| \leq 2\|P\|_{E \cap B\left(z_{0}, r\right)} \sum_{\mu=1}^{n} \frac{\prod_{\nu=1, v \neq \mu}^{n}\left|z_{0}-z_{\nu}\right|}{\prod_{\nu=0, v \neq \mu}^{n}\left|z_{\mu}-z_{\nu}\right|} .
$$

Since $\left|z_{\mu}-z_{v}\right| \geq\left|t_{\mu}-t_{v}\right|$ for each $\mu$ and $v$, by inequality (7), we have

$$
\begin{gathered}
\left|P^{\prime}\left(z_{0}\right)\right| \leq 2\|P\|_{E \cap B\left(z_{0}, r\right)} \sum_{\mu=1}^{n} \frac{\prod_{\nu=1, v \neq \mu}^{n} t_{\nu}}{\prod_{\nu=0, \nu \neq \mu}^{n}\left|t_{\mu}-t_{\nu}\right|}=\left.2\|P\|\right|_{E \cap B\left(z_{0}, r\right)} \sum_{\mu=1}^{n}\left|L_{\mu}^{\prime}(0)\right| \\
\quad \leq 2\|P\|_{E \cap B\left(z_{0}, r\right)} \sum_{\mu=1}^{n} C_{n}\left\|L_{\mu}\right\|_{T}=2 n C_{n}\|P\|_{E \cap B\left(z_{0}, r\right)} .
\end{gathered}
$$

Proposition 2.1 and its generalizations ([4, Theorems 1.1 and 1.2]) seem to be a result of independent interest (see [4, Section 3]).

Note that inequality (8) does not imply property (7). It is sufficient to consider $E:=\{0\} \cup\{z \in \mathbb{C}:|z|=r\}$ and $z_{0}=0$. By Cauchy's inequality, condition (8) is satisfied but $T=\{0, r\}$ does not admit any Markov inequality.

The next proposition shows that in the definition of the local Markov property we can replace $P^{(j)}\left(z_{0}\right)$ by $P^{\prime}\left(z_{0}\right)$.

Proposition 2.2 If $E \subset \mathbb{C}$ is a compact set, $m, \kappa \geq 1$ and there exists $c_{1}>0$ such that for every $z_{0} \in E, r \in(0,1], P \in \mathcal{P}_{n}(\mathbb{C})$

$$
\left|P^{\prime}\left(z_{0}\right)\right| \leq \frac{c_{1} n^{\kappa}}{r^{m}}\|P\|_{E \cap B\left(z_{0}, r\right)}
$$

then the set $E$ admits the $\operatorname{LMP}(m, \kappa+m)$.

Proof Fix arbitrary $n \in \mathbb{N}, z_{0}, r, P$ and $j \in\{2, \ldots, n\}$. By applying the assumption to the derivative $P^{(j-1)}$ and radius $r / n$, we see that

$$
\left|P^{(j)}\left(z_{0}\right)\right| \leq \frac{c_{1} n^{m+\kappa}}{r^{m}}\left\|P^{(j-1)}\right\|_{E \cap B\left(z_{0}, r / n\right)} .
$$

Let $z_{1}$ be a point of $E \cap B\left(z_{0}, \frac{r}{n}\right)$ such that $\left\|P^{(j-1)}\right\|_{E \cap B\left(z_{0}, r / n\right)}=\left|P^{(j-1)}\left(z_{1}\right)\right|$. Next we obtain similarly

$$
\left|P^{(j)}\left(z_{0}\right)\right| \leq \frac{c_{1} n^{m+\kappa}}{r^{m}}\left|P^{(j-1)}\left(z_{1}\right)\right| \leq\left(\frac{c_{1} n^{m+\kappa}}{r^{m}}\right)^{2}\left\|P^{(j-2)}\right\|_{E \cap B\left(z_{1}, r / n\right)} .
$$


We continue in this fashion to obtain points $z_{1}, \ldots, z_{j-1}$ such that for all $v=1, \ldots, j-$ 1 we have $z_{v} \in E \cap B\left(z_{v-1}, \frac{r}{n}\right)$ and ||$P^{(j-v)} \|_{E \cap B\left(z_{v-1}, \frac{r}{n}\right)}=\left|P^{(j-v)}\left(z_{v}\right)\right|$. This way we conclude that

$$
\begin{gathered}
\left|P^{(j)}\left(z_{0}\right)\right| \leq\left(\frac{c_{1} n^{m+\kappa}}{r^{m}}\right)^{j}\|P\|_{E \cap B\left(z_{j-1}, r / n\right)} \\
\leq\left(\frac{c_{1} n^{m+\kappa}}{r^{m}}\right)^{j}\|P\|_{E \cap B\left(z_{0}, j r / n\right)} \leq\left(\frac{c_{1} n^{m+\kappa}}{r^{m}}\right)^{j}\|P\|_{E \cap B\left(z_{0}, r\right)} .
\end{gathered}
$$

Lemma 2.3 If $P \in \mathcal{P}_{n}$ and $r>0$, then there exists an interval $I \subset[0, r]$ of length at least $\frac{r}{4 n^{2}}$ such that

$$
\|P\|_{[0, r]} \leq 2|P(x)| \quad \text { for all } x \in I
$$

Proof Let $x_{0}$ be a point of $[0, r]$ such that $\left|P\left(x_{0}\right)\right|=\|P\|_{[0, r]}$. Put $I:=\left[x_{0}-\frac{r}{4 n^{2}}\right.$, $\left.x_{0}+\frac{r}{4 n^{2}}\right] \cap[0, r]$ and consider an arbitrary point $x \in I$. The mean value theorem leads to

$$
\left|P\left(x_{0}\right)-P(x)\right| \leq\left\|P^{\prime}\right\|_{I} \cdot\left|x_{0}-x\right| \leq \frac{r}{4 n^{2}}\left\|P^{\prime}\right\|_{[0, r]} .
$$

The interval $[0, r]$ admits the classical Markov inequality (see e.g. [10, Ch.4, Theorem 1.4]), hence

$$
\left\|P^{\prime}\right\|_{[0, r]} \leq \frac{2 n^{2}}{r}\|P\|_{[0, r]}=\frac{2 n^{2}}{r}\left|P\left(x_{0}\right)\right|
$$

From the above it follows that $\left|P\left(x_{0}\right)\right|-|P(x)| \leq \frac{1}{2}\left|P\left(x_{0}\right)\right|$, and finally $\left|P\left(x_{0}\right)\right| \leq$ $2|P(x)|$.

Proof of Theorem 1.9.a Fix an arbitrary integer $a \geq m+1$ and put $m_{a}=\frac{s\left(a+c_{o}\right)-m}{a-m} \geq 1$ and $\kappa_{a}:=\frac{(\kappa+2 s)\left(a+c_{o}\right)}{a-m}>3$. We will use Proposition 2.2 to prove $\operatorname{LMP}\left(m_{a}, \kappa_{a}+m_{a}\right)$, which for sufficiently large $a$ leads to the assertion of the theorem, because $\lim _{a \rightarrow \infty} m_{a}=s$ and $\lim _{a \rightarrow \infty}\left(\kappa_{a}+m_{a}\right)=\kappa+3 s$.

Hence we need to prove the assumption of Proposition 2.2. For that purpose, fix arbitrarily $n \in \mathbb{N}, z_{0} \in E$ and $r \in(0,1]$. Define $T=T\left(z_{0}, r\right), t_{0}, \ldots, t_{n}$ and $L_{\mu}$ for $\mu=$ $1, \ldots, n$ as in Proposition 2.1. Note that the set $T\left(z_{0}, r\right)$ contains at least $n+1$ different points, because every compact admitting $\mathrm{KPH}$ is a perfect set and thus $z_{0}$ is not an isolated point of $E$ and $t_{0}=\min \left\{t_{0}, \ldots, t_{n}\right\}=0$ is not an isolated point of $T\left(z_{0}, r\right)$. Let $I_{\mu}$ be an interval of length at least $\frac{r}{4 n^{2}}$ constructed for the polynomial $L_{\mu}$ as in Lemma 2.3.

If for every $\mu=1, \ldots, n$ there exists $z_{\mu} \in E$ such that $\left|z_{\mu}-z_{0}\right| \in I_{\mu}$, then we use Proposition 2.1. Specifically, in this case for $\mu=1, \ldots, n$, the interval $I_{\mu}$ intersects the set $T\left(z_{0}, r\right)$, say at the point $\tau_{\mu}$. By applying the classic Markov inequality for the interval $[0, r]$ and Lemma 2.3, we obtain for $\mu=1, \ldots, n$

$$
\left|L_{\mu}^{\prime}(0)\right| \leq \frac{2 n^{2}}{r}\left\|L_{\mu}\right\|_{[0, r]} \leq \frac{4 n^{2}}{r}\left|L_{\mu}\left(\tau_{\mu}\right)\right| \leq \frac{4 n^{2}}{r}\left\|L_{\mu}\right\|_{T\left(z_{0}, r\right)} .
$$


Hence by Proposition 2.1, we have

$$
\left|P^{\prime}\left(z_{0}\right)\right| \leq \frac{8 n^{3}}{r}\|P\|_{E \cap B\left(z_{0}, r\right)} \leq \frac{8 n^{\kappa_{a}}}{r^{m_{a}}}\|P\|_{E \cap B\left(z_{0}, r\right)}
$$

for all polynomials $P \in \mathcal{P}_{n}$ as required in the assumption of Proposition 2.2.

We now turn to the case where $I_{\mu} \cap T\left(z_{0}, r\right)=\emptyset$ for some $\mu \in\{1, \ldots, n\}$, which implies that there is an empty annulus around $z_{0}$ of a certain minimum size. We shall have established the first implication in Theorem 1.9.a if we prove that in this case we have for all $P \in \mathcal{P}_{n}$

$$
\left|P^{\prime}\left(z_{0}\right)\right| \leq C n^{\kappa \frac{a+c_{o}}{a-m}}\left(\frac{r^{m}}{l_{\mu}^{S\left(a+c_{o}\right)}}\right)^{\frac{1}{a-m}}\|P\|_{E \cap B\left(z_{0}, r\right)},
$$

where $C$ is a constant independent of $P, n, r, z_{0}$ and $l_{\mu}$ is the length of $I_{\mu}$. Indeed, by Lemma $2.3, l_{\mu} \geq \frac{r}{4 n^{2}}$ and applying this to inequality (10) we obtain

$$
\left|P^{\prime}\left(z_{0}\right)\right| \leq C n^{\kappa \frac{a+c_{o}}{a-m}} \frac{\left(4 n^{2}\right)^{\frac{s\left(a+c_{o}\right)}{a-m}}}{r^{\frac{s\left(a+c_{o}\right)-m}{a-m}}}\|P\|_{E \cap B\left(z_{0}, r\right)}=4^{s \frac{a+c_{o}}{a-m}} C \frac{n^{\kappa_{a}}}{r^{m_{a}}}\|P\|_{E \cap B\left(z_{0}, r\right)},
$$

as required.

It remains to show inequality (10) for all $P \in \mathcal{P}_{n}$. For this purpose, we write $\left[\rho_{0}, \rho_{1}\right]$ for the interval $I_{\mu}$. Of course, $0<\rho_{0}<\rho_{1} \leq r$ and $\rho_{1}-\rho_{0}=l_{\mu}$.

The rest of the proof is adapted from [Bos and Milman (A Geometric Interpretation and the Equality of Exponents in Markov and Gagliardo-Nirenberg (Sobolev) Type Inequalities for Singular Compact Domains (preprint)), Theorem A]. Let $\varepsilon \in \mathcal{C}^{\infty}(\mathbb{R})$ be any cutoff function with the following properties:

(a) $0 \leq \varepsilon(x) \leq 1$ for all $x \in \mathbb{R}$,

(b) $\varepsilon(x)=1$ for $x \leq \frac{1}{3}$,

(c) $\varepsilon(x)=0$ for $x \geq \frac{2}{3}$

and put $h(z):=\varepsilon\left(\frac{\left|z-z_{0}\right|-\rho_{0}}{l_{\mu}}\right)$ so that $h \in \mathcal{C}^{\infty}(\mathbb{C})$.

Now fix arbitrarily $P \in \mathcal{P}_{n}$ and let $Q(z):=\left(P(z)-P\left(z_{0}\right)\right)^{a}, Q \in \mathcal{P}_{a n}$, and $f(z):=$ $h(z) Q(z)$. Choose any $l \in \mathbb{N}$ such that $a^{2} n \leq l \leq 2 a^{2} n$. Since $T\left(z_{0}, r\right) \cap\left[\rho_{0}, \rho_{0}+l_{\mu}\right]=$ $\emptyset$, we have $f \in \mathcal{H}^{\infty}\left(E_{\delta}\right)$, where $\delta:=\frac{l_{\mu}}{3} \leq \frac{r}{3}$.

We see that

$$
\begin{gathered}
\|f\|_{E}=\|f\|_{E \cap B\left(z_{0}, \rho_{1}\right)} \leq\|Q\|_{E \cap B\left(z_{0}, \rho_{1}\right)} \leq\|Q\|_{E \cap B\left(z_{0}, r\right)}, \\
\|f\|_{E_{\delta}}=\|f\|_{E_{\delta} \cap B\left(z_{0}, \rho_{1}\right)} \leq\|f\|_{B\left(z_{0}, \rho_{1}\right)} \leq\|Q\|_{B\left(z_{0}, \rho_{1}\right)} \leq\|Q\|_{B\left(z_{0}, r\right)},
\end{gathered}
$$

and by the $\operatorname{KPH}(m, s, \kappa)$, we have for arbitrary $j \in \mathbb{N}$ such that $j \leq a n \leq \frac{l}{a}<\frac{l}{m}$

$$
\left|Q^{(j)}\left(z_{0}\right)\right| \leq\left\|\frac{\partial^{j} f}{\partial z^{j}}\right\|_{E}=|f|_{E, j} \leq\left(\frac{c_{1} l^{\kappa}}{\delta^{s}}\right)^{j+c_{o}}\|f\|_{E}^{1-\frac{m j}{T}}\|f\|_{E_{\delta}}^{\frac{m j}{l}} .
$$

From inequalities (11-13) and the choice of $\delta$, it follows that

$$
\left|Q^{(j)}\left(z_{0}\right)\right| \leq\left(\frac{3^{s} c_{1} l^{\kappa}}{l_{\mu}^{s}}\right)^{j+c_{o}}\|Q\|_{E \cap B\left(z_{0}, r\right)}^{1-\frac{m j}{l}}\|Q\|_{B\left(z_{0}, r\right)}^{\frac{m j}{l}}
$$


Our next objective is to estimate

$$
\lambda:=\left(\frac{\|Q\|_{B\left(z_{0}, r\right)}}{\|Q\|_{E \cap B\left(z_{0}, r\right)}}\right)^{\frac{1}{a n}} \geq 1 .
$$

By inequality (14), we have for any $j=1, \ldots$, an

$$
\left|Q^{(j)}\left(z_{0}\right)\right| \leq\left(\frac{3^{s} c_{1} l^{\kappa}}{l_{\mu}^{s}}\right)^{j+c_{o}} \lambda^{\frac{m j}{a}}\|Q\|_{E \cap B\left(z_{0}, r\right)}
$$

and for $j=0$ this is trivially true too. From this, applying Taylor's formula and the fact that $l_{\mu} \leq r \leq 1$, we get

$$
\begin{aligned}
& \|Q\|_{B\left(z_{0}, r\right)} \leq \sum_{j=0}^{a n} \frac{r^{j}\left|Q^{(j)}\left(z_{0}\right)\right|}{j !} \leq \sum_{j=0}^{a n}\left(3^{s} c_{1} l^{\kappa}\right)^{j+c_{o}}\left(\frac{r}{l_{\mu}^{s}}\right)^{j} \frac{\lambda^{\frac{m j}{a}}}{l_{\mu}^{s c_{o}}}\|Q\|_{E \cap B\left(z_{0}, r\right)} \\
& \leq\left(2 \cdot 3^{s} c_{1} l^{\kappa}\right)^{a n+c_{o}}\left(\frac{r}{l_{\mu}^{s}}\right)^{a n} \frac{\lambda^{m n}}{l_{\mu}^{s c_{o}}}\|Q\|_{E \cap B\left(z_{0}, r\right)} .
\end{aligned}
$$

It follows that

$$
\lambda^{a n}=\frac{\|Q\|_{B\left(z_{0}, r\right)}}{\|Q\|_{E \cap B\left(z_{0}, r\right)}} \leq\left(2 \cdot 3^{s} c_{1} l^{\kappa}\right)^{a n+c_{o}}\left(\frac{r}{l_{\mu}^{s}}\right)^{a n} \frac{\lambda^{m n}}{l_{\mu}^{s c_{o}}},
$$

and consequently

$$
\lambda \leq\left(2 \cdot 3^{s} c_{1} l^{\kappa}\right)^{\frac{a+c_{o} / n}{a-m}}\left(\frac{r}{l_{\mu}^{s}}\right)^{\frac{a}{a-m}} \cdot\left(\frac{1}{l_{\mu}^{s c_{o}}}\right)^{\frac{1}{(a-m) n}} .
$$

By combining this estimate with inequality (15), we can assert that

$$
\begin{aligned}
\left|Q^{(j)}\left(z_{0}\right)\right| & \leq\left(2 \cdot 3^{s} c_{1} l^{\kappa}\right)^{j+c_{o}+\frac{m j\left(a+\frac{c o}{n}\right)}{a(a-m)}}\left(\frac{1}{l_{\mu}}\right)^{s\left(j+c_{o}\right)+\frac{j m s}{a-m}+\frac{s c_{o} m j}{a n(a-m)}} r^{\frac{j m}{a-m}}\|Q\|_{E \cap B\left(z_{0}, r\right)} \\
& \leq\left(2 \cdot 3^{s} c_{1} l^{\kappa}\right)^{\frac{a}{a-m}\left(j+c_{o}\right)}\left(\frac{1}{l_{\mu}}\right)^{\frac{a s}{a-m}\left(j+c_{o}\right)} r^{\frac{j m}{a-m}}\|Q\|_{E \cap B\left(z_{0}, r\right)},
\end{aligned}
$$

because $\frac{m j}{a}\left(a+\frac{c_{o}}{n}\right)=m\left(j+\frac{j}{a n} c_{o}\right) \leq m\left(j+c_{o}\right)$ and $(a-m) s\left(j+c_{o}\right)+j m s+s c_{o} m \frac{j}{a n} \leq$ $(a-m) s\left(j+c_{o}\right)+j m s+s c_{o} m=a s\left(j+c_{o}\right)$. Specifically, for $j=a$ we have

$$
a !\left|P^{\prime}\left(z_{0}\right)\right|^{a}=\left|Q^{(a)}\left(z_{0}\right)\right| \leq\left(2 \cdot 3^{s} c_{1} l^{\kappa}\right)^{a \frac{a+c_{0}}{a-m}}\left(\frac{r^{m}}{l_{\mu}^{s\left(a+c_{o}\right)}}\right)^{\frac{a}{a-m}}\|Q\|_{E \cap B\left(z_{0}, r\right)} .
$$

By the definition of $Q$ and since $l \leq 2 a^{2} n$, we finally get

$$
\left|P^{\prime}\left(z_{0}\right)\right| \leq 2\left(2^{1+\kappa} 3^{s} a^{2 \kappa} c_{1} n^{\kappa}\right)^{\frac{a+c_{o}}{a-m}}\left(\frac{r^{m}}{l_{\mu}^{s\left(a+c_{o}\right)}}\right)^{\frac{1}{a-m}}\|P\|_{E \cap B\left(z_{0}, r\right)},
$$

which implies inequality (10).

The second statement of Theorem 1.9. a is easy to show because $m_{a}=1$ for $s=1$, $c_{o}=0$ and arbitrary $m$. 


\section{The LMP Implies the KPQ}

Lemma 3.1 If $E \subset \mathbb{C}$ is a compact set with the $\operatorname{LMP}(m, \kappa)$, then there exist $c_{1} \geq 1$ such that for every $z_{0} \in E$ and $l \in \mathbb{N}$

$$
\left|\frac{\partial^{j} g}{\partial z^{j}}\left(z_{0}\right)\right| \leq\left(c_{1} l^{\kappa}\right)^{j}\|g\|_{E}^{1-\frac{m j}{l}}\|g\|_{\operatorname{conv}(E), l}^{\frac{m j}{l}}, \quad j=1, . ., l
$$

for any function $g \in \mathcal{C}^{\infty}(\mathbb{C})$ that is $\bar{\partial}$-flat at $z_{0}$ and $g / E \not \equiv 0$.

Proof Our reasoning is a modification of that given by Bos and Milman (A Geometric Interpretation and the Equality of Exponents in Markov and GagliardoNirenberg (Sobolev) Type Inequalities for Singular Compact Domains (preprint)). Fix $z_{0} \in E, \quad l \in \mathbb{N}$ and $g \in \mathcal{C}^{\infty}(\mathbb{C})$ satisfying the assumptions of the lemma. Let $T_{z_{0}}^{l} g$ be the Taylor polynomial defined by $T_{z_{0}}^{l} g(z):=\sum_{|\alpha| \leq l-1} \frac{1}{\alpha !} D^{\alpha} g\left(z_{0}\right)\left(z-z_{0}\right)^{\alpha}=$ $\sum_{j=0}^{l-1} \frac{1}{j !} \frac{d^{j} g}{d z^{j}}\left(z_{0}\right)\left(z-z_{0}\right)^{j}$, because $g$ is $\bar{\partial}$-flat in $z_{0}$. By the local Markov property, it follows that

$$
\left|\frac{\partial^{j} g}{\partial z^{j}}\left(z_{0}\right)\right|=\left|\frac{d^{j}}{d z^{j}} T_{z_{0}}^{l} g\left(z_{0}\right)\right| \leq\left[\frac{c_{1}(l-1)^{\kappa}}{r^{m}}\right]^{j}\left\|T_{z_{0}}^{l} g\right\|_{E \cap B\left(z_{0}, r\right)}
$$

for every $r \in(0,1]$ and $j=1, \ldots, l-1$. If $R_{z_{0}}^{l} g:=g-T_{z_{0}}^{l} g$ is the Taylor remainder, then we have

$$
\begin{aligned}
\left|\frac{\partial^{j} g}{\partial z^{j}}\left(z_{0}\right)\right| & \leq\left(\frac{c_{1} l^{\kappa}}{r^{m}}\right)^{j}\left(\|g\|_{E \cap B\left(z_{0}, r\right)}+\left\|R_{z_{0}}^{l} g\right\|_{E \cap B\left(z_{0}, r\right)}\right) \\
& \leq\left(\frac{c_{1} l^{\kappa}}{r^{m}}\right)^{j}\left(\|g\|_{E}+r^{l}|g|_{\operatorname{conv}(E), l}\right)
\end{aligned}
$$

for $j=1, \ldots, l-1$ and this goes also for $j=l$, which is easy to check because $\left|\frac{\partial^{l} g}{\partial z^{l}}\left(z_{0}\right)\right| \leq|g|_{\operatorname{conv}(E), l \text {. Put }}$

$$
r=\left(\frac{\|g\|_{E}}{A}\right)^{\frac{1}{l}}, \quad \text { where } \quad A:=\|g\|_{E}+|g|_{\operatorname{conv}(E), l} .
$$

Of course, $r \in(0,1]$. From the above it follows that

$$
\begin{aligned}
\left|\frac{\partial^{j} g}{\partial z^{j}}\left(z_{0}\right)\right| & \leq\left(\frac{c_{1} l^{\kappa}}{\|g\|_{E}^{m / l}} A^{\frac{m}{l}}\right)^{j}\left(\|g\|_{E}+\frac{\|g\|_{E}}{A}|g|_{\operatorname{conv}(E), l}\right) \\
& =\left(c_{1} l^{\kappa}\right)^{j} A^{\frac{m j}{l}}\|g\|_{E}^{1-\frac{m j}{l}}\left(1+\frac{1}{A}|g|_{\operatorname{conv}(E), l}\right) .
\end{aligned}
$$

Since $|g|_{\operatorname{conv}(E), l}<A \leq \|\left. g\right|_{\operatorname{conv}(E), l}$, we conclude that

$$
\begin{aligned}
\left|\frac{\partial^{j} g}{\partial z^{j}}\left(z_{0}\right)\right| & \leq\left(c_{1} l^{\kappa}\right)^{j}\|g\|_{\operatorname{conv}(E), l}^{\frac{m j}{l}}\|g\|_{E}^{1-\frac{m j}{l}} \cdot 2 \\
& \leq\left(2 c_{1} l^{\kappa}\right)^{j}\|g\|_{E}^{1-\frac{m j}{l}}\|g\|_{\operatorname{conv}(E), l}^{\frac{m j}{l}}, \quad j \in\{1, \ldots, l\},
\end{aligned}
$$

and this is precisely the assertion of the lemma. 
Proof of Theorem 1.9.b We first observe that $E$ is perfect and thus $\mathcal{A}^{\infty}$-determining, i.e., if $f \in \mathcal{A}^{\infty}(E)$ and $f / E \equiv 0$ then $\left(D^{\alpha} f\right) / E \equiv 0$ for all $\alpha \in \mathbb{N}_{0}^{2}$.

Fix $f \in \mathcal{A}^{\infty}(E)$. We can certainly assume that $f / E \not \equiv 0$, since otherwise the inequality in KPQ would be fulfilled trivially. Consider an arbitrary function $g \in \mathcal{C}^{\infty}(\mathbb{C})$ that is $\bar{\partial}$-flat on $E$ and $g / E \equiv f /{ }_{E}$. Lemma 3.1 now gives

$$
\left\|\frac{\partial^{j} g}{\partial z^{j}}\right\|_{E} \leq\left(c_{1} l^{\kappa}\right)^{j}\|g\|_{E}^{1-\frac{m j}{l}}\|g\|_{\operatorname{conv}(E), l}^{\frac{m j}{l}} \quad \text { as } \quad 1 \leq j \leq l .
$$

We have $\frac{\partial^{j} g}{\partial z^{j}} / E \equiv \frac{\partial^{j} f}{\partial z^{j}} / E$, because $g-f \in \mathcal{A}^{\infty}(E),(g-f) / E \equiv 0$ and $E$ is $\mathcal{A}^{\infty}$ determining. Consequently, for $1 \leq j \leq l$ we get

$$
\left\|\frac{\partial^{j} f}{\partial z^{j}}\right\|_{E} \leq\left(c_{1} l^{\kappa}\right)^{j}\|f\|_{E}^{1-\frac{m j}{l}}\|f\|_{E, l}^{\frac{m j}{l}}
$$

which completes the proof.

\section{The KPQH Implies the KPH}

First we give a lemma on cutoff functions, which allows us to estimate the holomorphic quotient norms. The lemma is an adaptation to the complex case of a result given e.g. in [Bos and Milman (A Geometric Interpretation and the Equality of Exponents in Markov and Gagliardo-Nirenberg (Sobolev) Type Inequalities for Singular Compact Domains (preprint)), Lemma 4.12].

Lemma 4.1 For any compact set $E \subset \mathbb{C}$ and radius $\varepsilon \in(0,1]$ there exists a cutoff function $u \in \mathcal{C}^{\infty}(\mathbb{C})$ such that

(a) $0 \leq u(z) \leq 1$ for all $z \in \mathbb{C}$,

(b) $u(z)=1$ as $\operatorname{dist}(z, E) \leq \frac{\varepsilon}{8}$,

(c) $u(z)=0$ as $\operatorname{dist}(z, E) \geq \varepsilon$,

(d) $\left\|D^{\alpha} u\right\|_{\mathbb{C}} \leq \frac{C_{|\alpha|}}{\varepsilon^{|\alpha|}}$ for all $\alpha=\left(\alpha_{1}, \alpha_{2}\right) \in \mathbb{N}_{0}^{2}$,

where $C_{j}=d j^{4 j}$ for $j \in \mathbb{N}, C_{0}=1$ and $d \geq 1$ is some absolute constant.

Proposition 4.2 For any compact set $E \subset \mathbb{C}, \delta \in(0,1], f \in \mathcal{H}^{\infty}\left(E_{\delta}\right)$ and $l \in \mathbb{N}$ we have

$$
\left\langle\langle f\rangle_{E_{\delta / 17}, l} \leq \frac{(2 d l)^{5 l}}{\delta^{l}}\|f\|_{E_{\delta}},\right.
$$

where $d \geq 1$ is the absolute constant from Lemma 4.1 .

Proof Let $u \in \mathcal{C}^{\infty}(\mathbb{C})$ be the cutoff function given in Lemma 4.1 for the set $E$ and radius $\varepsilon=\frac{\delta}{2}$. We put $\widetilde{f}=u f$ and see that $\widetilde{f} \in \mathcal{H}^{\infty}\left(E_{\frac{\delta}{17}}\right)$ because $\widetilde{f} \equiv f$ on $E_{\frac{\delta}{16}}$, which contains an open neighbourhood of the set $E_{\frac{\delta}{17}}$. In $\mathbb{C} \backslash E_{\frac{\delta}{2}}$ we have $u \equiv 0$ and hence $D^{\alpha} \tilde{f} \equiv 0$ for all $\alpha \in \mathbb{N}_{0}^{2}$. Consequently, by the definition of the holomorphic quotient 
norms, we have $\left\langle\langle f\rangle_{E_{\delta / 17}, l} \leq\|\tilde{f}\|_{\operatorname{conv}\left(E_{\delta / 17}\right), l} \leq\|\tilde{f}\|_{\mathbb{C}, l}=\|\tilde{f}\|_{E_{\delta / 2}, l}\right.$. By the Leibniz rule, we obtain for every $z \in E_{\delta / 2}$ and $\alpha \in \mathbb{N}_{0}^{2}$

$$
D^{\alpha} \tilde{f}(z)=\sum_{\beta \leq \alpha}\left(\begin{array}{l}
\alpha \\
\beta
\end{array}\right) D^{\beta} u(z) D^{\alpha-\beta} f(z)=\sum_{\beta_{1} \leq \alpha_{1}}\left(\begin{array}{c}
\alpha_{1} \\
\beta_{1}
\end{array}\right) D^{\left(\beta_{1}, \alpha_{2}\right)} u(z) f^{\left(\alpha_{1}-\beta_{1}\right)}(z)
$$

because $f$ is holomorphic in $E_{\delta}$. As usual, $\beta \leq \alpha$ means that $\beta_{1} \leq \alpha_{1}, \beta_{2} \leq \alpha_{2}$ and $\left(\begin{array}{l}\alpha \\ \beta\end{array}\right)=$ $\left(\begin{array}{l}\alpha_{1} \\ \beta_{1}\end{array}\right)\left(\begin{array}{l}\alpha_{2} \\ \beta_{2}\end{array}\right)$. The properties of the cutoff function and Cauchy's integral formula lead us to

$$
\begin{aligned}
& \left\|D^{\alpha} \tilde{f}\right\|_{E_{\frac{\delta}{2}}} \leq \sum_{\beta_{1} \leq \alpha_{1}}\left(\begin{array}{c}
\alpha_{1} \\
\beta_{1}
\end{array}\right) \frac{C_{\beta_{1}+\alpha_{2}}}{(\delta / 2)^{\beta_{1}+\alpha_{2}}} \frac{\left(\alpha_{1}-\beta_{1}\right) !}{(\delta / 2)^{\alpha_{1}-\beta_{1}}}\|f\|_{E_{\delta}} \\
& =\sum_{\beta_{1} \leq \alpha_{1}} \frac{\alpha_{1} !}{\beta_{1} !} \frac{C_{\beta_{1}+\alpha_{2}}}{(\delta / 2)^{|\alpha|}}\|f\|_{E_{\delta}} \leq \frac{e l ! C_{l}}{(\delta / 2)^{l}}\|f\|_{E_{\delta}} \leq \frac{2 e l^{l} C_{l}}{\delta^{l}}\|f\|_{E_{\delta}}=\frac{2 e d l^{5 l}}{\delta^{l}}\|f\|_{E_{\delta}}
\end{aligned}
$$

for $|\alpha|=l$. Finally, we see that

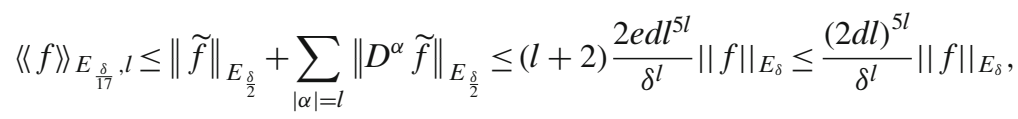

because $(l+2) 2 e \leq 2^{5 l}$.

Proof of Theorem 1.9.d Fix $j, l \in \mathbb{N}$ such that $l \geq m j, \delta \in(0,1]$ and $f \in \mathcal{H}^{\infty}\left(E_{\delta}\right)$. We combine KPQH with Proposition 4.2 to obtain

$$
\begin{aligned}
& |f|_{E, j} \leq\left(\frac{c_{1} l^{\kappa}}{(\delta / 17)^{s}}\right)\|f\|_{E}^{1-\frac{m j}{l}}\langle f f\rangle_{E_{\delta / 1}, l}^{\frac{m j}{l}} \leq\left(\frac{17^{s} c_{1} l^{\kappa}}{\delta^{s}}\right)\|f\|_{E}^{1-\frac{m j}{l}} \frac{(2 d l)^{5 m j}}{\delta^{m j}}\|f\|_{E_{\delta}}^{\frac{m j}{l}} \\
& =\left(\frac{17^{s} c_{1} l^{\kappa}(2 d l)^{5 m}}{\delta^{m+s}}\right)^{j}\|f\|_{E}^{1-\frac{m j}{l}}\|f\|_{E_{\delta}}^{\frac{m j}{l}}=\left(\frac{\widetilde{c}_{1} l^{\kappa+5 m}}{\delta^{m+s}}\right)^{j}\|f\|_{E}^{1-\frac{m j}{l}}\|f\|_{E_{\delta}}^{\frac{m j}{l}},
\end{aligned}
$$

where $\widetilde{c}_{1}=17^{s} c_{1}(2 d)^{5 m}$. This proves Theorem 1.9.d.

It is worth noticing that if we replace in Definitions $1.4,1.5,1.7$ and $1.8 c_{1} n^{\kappa}$ or $c_{1} l^{\kappa}$ by $c_{n}$ or $c_{l}$ respectively, then Theorem 1.9. remains true. Bos and Milman labeled such properties of Markov or Sobolev type in the real case as weak inequalities.

\section{The LMP( $m$ ) Implies $(m, \infty)$-perfectness}

Definition 5.1 (see [3, Definition 3.1] for more details) Let $m \geq 1$ and $k \in\{2$, $3,4, \ldots\}$. A compact set $E \subset \mathbb{C}$ is $(m, k)$-perfect if there exist constants $a, b>$ 0 such that for every $z \in E$ we can construct a family of closed $\operatorname{discs}\{B\} \cup$ $\left\{B_{i_{1}, \ldots, i_{n}}\right\}_{n \in \mathbb{N}, i_{1}, \ldots, i_{n} \in\{1, \ldots, k\}}$ satisfying the following conditions

(i) $B_{i_{1}, \ldots, i_{n+1}} \subset B_{i_{1}, \ldots, i_{n}}$ for $n \in \mathbb{N}, i_{1}, \ldots, i_{n+1}=1,2, \ldots, k$, $B_{i_{1}} \subset B$ for $i_{1}=1,2, \ldots, k$, 
(ii) $\operatorname{diam} B_{i_{1}, \ldots, i_{n+1}}=a\left(\operatorname{diam} B_{i_{1}, \ldots, i_{n}}\right)^{m}$ for $i_{1}, \ldots, i_{n+1}=1,2, \ldots, k$, $\operatorname{diam} B_{i_{1}}=a(\operatorname{diam} B)^{m}=a$ for $i_{1}=1,2, \ldots, k$

(iii) $\operatorname{dist}\left(B_{i_{1}, \ldots i_{n}, p}, B_{i_{1}, \ldots, i_{n}, q}\right) \geq b\left(\operatorname{diam} B_{i_{1}, \ldots, i_{n}}\right)^{m} \quad$ for $\quad n \in \mathbb{N}, \quad i_{1}, \ldots, i_{n}, p, q=$ $1,2, \ldots, k, p \neq q$, $\operatorname{dist}\left(B_{p}, B_{q}\right) \geq b(\operatorname{diam} B)^{m}=b$ for $p, q=1,2, \ldots, k, p \neq q$,

(iv) $z \in \bigcap_{n=1}^{\infty} \bigcup_{i_{1}, \ldots, i_{n}=1}^{k} B_{i_{1}, \ldots, i_{n}} \subset E$,

where diam $D$ is the diameter of a set $D$ and $\operatorname{dist}\left(D_{1}, D_{2}\right)$ is the distance between sets $D_{1}, D_{2}$.

We have shown in [3, Theorem 3.4] that if $E$ is an $m$-perfect set then it is $(m, 2)$-perfect and if $E$ is $(m, k)$-perfect for some $k \in\{2,3, \ldots\}$ then it is $m^{2}$-perfect. Furthermore, an $(m, k)$-perfect set $E$ with $m<k$ satisfies the following estimate of the logarithmic capacity: there exists a constant $c>0$ such that

$$
\operatorname{cap}(E \cap B(z, r)) \geq c r^{\frac{k-1}{k-m} m^{2}}
$$

for every $z \in E$ and $r \in(0,1]$ (see [3, Proposition 3.6]).

Definition 5.2 We call a compact set $(m, \infty)$-perfect if it is $(m, k)$-perfect for every $k \in\{2,3, \ldots\}$.

According to the above, by the Wiener criterion (see e.g. [15, Theorem 5.4.1]), every $(m, k)$-perfect set with $m<k$ is L-regular. Therefore, $(m, \infty)$-perfectness implies L-regularity.

Theorem 5.3 If $m \geq 1$ and $E$ is a compact subset of the complex plane satisfying the following Markov property: for each $n \in \mathbb{N}$ there exists a constant $c_{n} \geq 1$ such that

$$
\left|P^{\prime}\left(z_{0}\right)\right| \leq \frac{c_{n}}{r^{m}}\|P\|_{E \cap B\left(z_{0}, r\right)}
$$

for all $z_{0} \in E, r \in(0,1]$ and $P \in \mathcal{P}_{n}(\mathbb{C})$, then $E$ is an $(m, \infty)$-perfect set.

Proof Fix $z_{0} \in E, r \in(0,1]$ and $k \in\{3,4, \ldots\}$. We will show that there exists a positive constant $a_{k}$ independent of $z_{0}, r$ and there exist points $z_{1}, \ldots, z_{k-1} \in E \cap B\left(z_{0}, r\right)$ such that

$$
\left|z_{i}-z_{j}\right| \geq a_{k} r^{m} \quad \text { for each } i, j \in\{0,1, \ldots, k-1\}, i \neq j
$$

which, according to [3, Lemma 3.2], is sufficient for $(m, k)$-perfectness. Note that $(m, 3)$-perfectness implies $(m, 2)$-perfectness.

Put $a_{k}:=\left[2(2 e)^{m}(k-1)^{m} c_{k-1}\right]^{-1}$. We construct the points $\left\{z_{j}\right\}_{j=1, \ldots, k-1}$ as follows. Let $\mu=1$. Put

$$
r_{\mu}:=r\left(\frac{k-2}{k-1}\right)^{\mu-1}
$$


Now find Fekete extremal points of order $k-1$ (see the beginning of Section 2) for the intersection $E \cap B\left(z_{0}, r_{\mu}\right)$ and denote them by $y_{1}^{(\mu)}, \ldots, y_{k}^{(\mu)}$. Let $L_{i}^{(\mu)}$ be the Lagrange polynomials associated with $y_{1}^{(\mu)}, \ldots, y_{k}^{(\mu)}$

$$
L_{i}^{(\mu)}(z)=\frac{\prod_{l=1, l \neq i}^{k}\left(z-y_{l}^{(\mu)}\right)}{\prod_{l=1, l \neq i}^{k}\left(y_{i}^{(\mu)}-y_{l}^{(\mu)}\right)}, \quad i=1, \ldots, k .
$$

Observe that for all $1 \leq i<j \leq k$

$$
\frac{1}{\left|y_{j}^{(\mu)}-y_{i}^{(\mu)}\right|^{2}}=\left|\frac{d}{d z} L_{i}^{(\mu)}\left(y_{j}^{(\mu)}\right)\right| \cdot\left|\frac{d}{d z} L_{j}^{(\mu)}\left(y_{i}^{(\mu)}\right)\right| .
$$

Thus, by inequality $(17)$, for every $\varrho \in(0,1]$

$$
\frac{1}{\left|y_{j}^{(\mu)}-y_{i}^{(\mu)}\right|^{2}} \leq\left(\frac{c_{k-1}}{\varrho^{m}}\right)^{2}\left\|L_{i}^{(\mu)}\right\|_{E \cap B\left(y_{j}^{(\mu)}, \varrho\right)}\left\|L_{j}^{(\mu)}\right\|_{E \cap B\left(y_{i}^{(\mu)}, \varrho\right)} .
$$

Assume that for each $i=1, \ldots, k$ we have

$$
\left|y_{i}^{(\mu)}-z_{0}\right| \leq \frac{k-\frac{3}{2}}{k-1} r_{\mu} .
$$

In that case we put $\varrho=\frac{1 / 2}{k-1} r_{\mu}$ and in consequence we conclude

$$
E \cap B\left(y_{i}^{(\mu)}, \varrho\right) \subset E \cap B\left(z_{0}, r_{\mu}\right) \quad \text { for every } i=1, \ldots, k
$$

and

$$
\begin{aligned}
\frac{1}{\left|y_{j}^{(\mu)}-y_{i}^{(\mu)}\right|^{2}} & \leq\left[\frac{c_{k-1}}{\left(\frac{1 / 2}{k-1} r_{\mu}\right)^{m}}\right]^{2}\left\|L_{i}^{(\mu)}\right\|_{E \cap B\left(z_{0}, r_{\mu}\right)}\left\|L_{j}^{(\mu)}\right\|_{E \cap B\left(z_{0}, r_{\mu}\right)} \\
& =\left[\frac{2^{m}(k-1)^{m} c_{k-1}}{r_{\mu}^{m}}\right]^{2} \quad \text { for all } 1 \leq i<j \leq k,
\end{aligned}
$$

because the norm of Lagrange polynomials on the set $E \cap B\left(z_{0}, r_{\mu}\right)$ equals 1 . Finally

$$
\left|y_{j}^{(\mu)}-y_{i}^{(\mu)}\right| \geq \frac{r_{\mu}^{m}}{2^{m}(k-1)^{m} c_{k-1}}=\frac{\left[r\left(\frac{k-2}{k-1}\right)^{\mu-1}\right]^{m}}{2^{m}(k-1)^{m} c_{k-1}} .
$$

Note that for all $x>0$ we have $\left(\frac{x+1}{x}\right)^{x}=\left(1+\frac{1}{x}\right)^{x}<e$ and in particular, putting $x:=$ $k-2$, we obtain $\left(\frac{k-2}{k-1}\right)^{k-2}>\frac{1}{e}$. From this it follows that for all $1 \leq i<j \leq k$

$$
\left|y_{j}^{(\mu)}-y_{i}^{(\mu)}\right|>\frac{r^{m}}{(2 e)^{m}(k-1)^{m} c_{k-1}}=2 a_{k} r^{m}, \quad \text { as long as } \mu \leq k-1 .
$$

Thus at most one point of the set $\left\{y_{i}^{(\mu)}\right\}_{i=1, \ldots, k}$ can be included in the interior of $B\left(z_{0}, a_{k} r^{m}\right)$. After removing from $\left\{y_{i}^{(\mu)}\right\}_{i=1, \ldots, k}$ that one point (or any arbitrary point 
if none belongs to the interior of $\left.B\left(z_{0}, a_{k} r^{m}\right)\right)$ we are left with $k-1$ points that meet the requirements of Lemma 3.2 in [3].

If assumption (19) is not met then we conclude that for a certain $i \in\{1, \ldots, k\}$ we have $\left|y_{i}^{(\mu)}-z_{0}\right|>\frac{k-3 / 2}{k-1} r_{\mu}$. We put $z_{\mu}:=y_{i}^{(\mu)}$ after which we increase $\mu$ by 1 and we return to inequality (18).

This way, either for a certain $\mu \in\{1, \ldots, k-1\}$ condition (19) will be satisfied and then the problem will be solved or we end up with a set of points $\left\{z_{\mu}\right\}_{\mu=1, \ldots, k-1} \subset E$ with the following property

$$
r\left(\frac{k-2}{k-1}\right)^{\mu-1} \frac{k-\frac{3}{2}}{k-1}=\frac{k-\frac{3}{2}}{k-1} r_{\mu}<\left|z_{\mu}-z_{0}\right| \leq r_{\mu}=r\left(\frac{k-2}{k-1}\right)^{\mu-1}
$$

for each $\mu \in\{1, \ldots, k-1\}$. From this it is easy to see that for all $1 \leq \mu<v \leq k-1$

$$
\begin{aligned}
& \left|z_{\mu}-z_{\nu}\right| \geq\left|z_{\mu}-z_{0}\right|-\left|z_{\nu}-z_{0}\right| \geq r\left(\frac{k-2}{k-1}\right)^{\mu-1} \frac{k-\frac{3}{2}}{k-1}-r\left(\frac{k-2}{k-1}\right)^{\nu-1} \\
& \quad \geq r\left(\frac{k-2}{k-1}\right)^{\mu-1}\left(\frac{k-\frac{3}{2}}{k-1}-\frac{k-2}{k-1}\right)
\end{aligned}
$$

hence

$$
\left|z_{\mu}-z_{\nu}\right| \geq \frac{r}{2 e(k-1)} \geq a_{k} r^{m}
$$

since $m \geq 1$ and $c_{k} \geq 1$. But also

$$
\left|z_{\mu}-z_{0}\right| \geq r\left(\frac{k-2}{k-1}\right)^{\mu-1} \frac{k-\frac{3}{2}}{k-1} \geq \frac{r}{2 e(k-1)} \geq a_{k} r^{m}
$$

for each $\mu=1, \ldots, k-1$, which finishes the proof.

The Markov property defined by inequality (17) can be found in $[3,8,20]$ Bos and Milman (A Geometric Interpretation and the Equality of Exponents in Markov and Gagliardo-Nirenberg (Sobolev) Type Inequalities for Singular Compact Domains (preprint)). If a set $E \subset \mathbb{C}$ has the $\operatorname{LMP}(m, \kappa)$ with constant $c_{1}$, then $c_{n}$ in inequality (17) equals $c_{1} n^{\kappa}$ and in the proof of Theorem 4.3 we can put $a_{k}=\frac{c^{\prime}}{k^{m+\kappa}}$ with some constant $c^{\prime}$ independent of $z_{0} \in E, r \in(0,1]$ and $k \in\{2,3, \ldots\}$.

Remark 5.4 By Theorem 5.3, every compact set $E \subset \mathbb{C}$ with the $\operatorname{LMP}(m)$ is $(m, \infty)$ perfect and, by consequence, it is L-regular. In this fashion, the L-regularity of sets with the LMP is proved in a different way than in [3] (compare estimate (16) with [3, Proposition 2.1]).

Remark 5.5 Let $E \subset \mathbb{C}$ be an $(m, \infty)$-perfect set. From what has already been described at the beginning of this section, we see that $E$ is L-regular, i.e. the Green function $g_{E}$ of the unbounded component of $\widehat{\mathbb{C}} \backslash E$ with the pole at $\infty$ is continuous. One may ask whether the function $g_{E}$ has the following Hölder continuity property

$$
g_{E}(z) \leq M[\operatorname{dist}(z, E)]^{s}, \quad \text { as } \operatorname{dist}(z, E) \leq 1,
$$


the constants $M, s>0$ being independent of $z$. The answer is negative. Indeed, consider the set

$$
E:=\{0\} \cup \bigcup_{l=2}^{\infty}\left[a_{l}, b_{l}\right], \quad \text { where } b_{l}:=\frac{1}{l !}, a_{l}:=\frac{l}{(l+1) !} \text { for } l=2,3, \ldots
$$

(compare with [12]).

Proposition 5.6 The set E constructed above is an $(m, \infty)$-perfect set for any $m>1$ and $E$ does not have the Hölder continuity property.

Proof The lack of the Hölder continuity property of $E$ is due to Goncharov and Uzun (Markov's property of compact sets in R (preprint)). Let us only outline the proof of the $(m, \infty)$-perfectness of $E$. By [3, Lemma 3.2], it is sufficient to find $a>0$ for fixed $m>0$ and $k \in \mathbb{N}$ such that for every $l \in \mathbb{N}$

$$
b_{l+1}-a_{l+1}>a a_{l}^{m} k .
$$

Thus we only need to show that there exists $a>0$ such that

$$
0<a<\frac{(l+1) !^{m}}{l^{m}(l+2) ! k} .
$$

Since $\mathrm{m}>1$, the assertion follows.

Open Access This article is distributed under the terms of the Creative Commons Attribution License which permits any use, distribution, and reproduction in any medium, provided the original author(s) and the source are credited.

\section{References}

1. Baran, M., Pleśniak, W.: Characterization of compact subsets of algebraic varieties in terms of Bernstein-type inequalities. St. Math. 141(3), 221-234 (2000)

2. Bialas-Ciez, L.: Markov Sets in $\mathbb{C}$ are not Polar. Bull. Pol. Acad. Sci., Math. 46(1), 83-89 (1998)

3. Bialas-Ciez, L., Eggink, R.: L-regularity of Markov sets and of $m$-perfect sets in the complex plane. Constr. Approx. 27, 237-252 (2008)

4. Bialas-Ciez, L., Kosek, M.: How to construct totally disconnected Markov sets? Ann. Mat. Pura Appl. 190(2), 209-224 (2011)

5. Bos, L., De Marchi, S., Sammariva, A., Vianello, M.: Computing multivariate Fekete and Leja points by numerical linear algebra. SIAM J. Numer. Anal. 48, 1984-1999 (2010)

6. Bos, L., Levenberg, N., Milman, P., Taylor, B.A.: Tangential Markov Inequalities Characterize Algebraic Submanifolds of $\mathbb{R}^{N}$. Indiana Univ. Math. J. 44(1), 115-138 (1995)

7. Bos, L.P., Milman, P.D.: On Markov and Sobolev type inequalities on sets in $\mathbb{R}^{n}$. In: Topics in Polynomials in One and Several Variables and Their Applications, pp. 81-100. World Sci. Publishing, River Edge, NJ (1993)

8. Bos, L.P., Milman, P.D.: Sobolev-Gagliardo-Nirenberg and Markov type inequalities on subanalytic domains. Geom. Func. Anal. 5(6), 853-923 (1995)

9. Calvi, J.-P., Levenberg, N.: Uniform approximation by discrete least squares polynomials. J. Approx. Theory 152(1), 82-100 (2008)

10. DeVore, R., Lorentz, G.: Constructive Approximation. Springer, Berlin Heidelberg (1993)

11. Eggink, R.: Markov's inequality in the complex plane. Master of Science Degree Memoir, Jagiellonian University, Kraków (in Polish) (1994)

12. Goncharov, A.: A compact set without Markov's property but with an extension operator for $\mathcal{C}^{\infty}$-functions. St. Math. 119(1), 27-35 (1996)

13. Klimek, M.: Pluripotential Theory. Oxford Science Publications, Oxford New York Tokyo (1991) 
14. Pleśniak, W.: Markov's inequality and the existence of an extension operator for $C^{\infty}$ functions. J. Approx. Theory 61, 106-117 (1990)

15. Ransford, T.: Potential Theory in the Complex Plane. In: Lond. Math. Soc. Stud. Texts, vol. 28. Cambridge (1995)

16. Siciak, J.: Extremal plurisubharmonic functions in $\mathbb{C}^{N}$. Ann. Pol. Math. 39, 175-211 (1981)

17. Siciak, J.: Highly noncontinuable functions on polynomially convex sets. Univ. Iagello. Acta Math. 25, 95-107 (1985)

18. Siciak, J.: Rapid polynomial approximation on compact sets in $\mathbb{C}^{n}$, Univ. Iagello. Acta Math. 30, 145-154 (1993)

19. Siciak, J.: Compact sets in $\mathbf{R}^{n}$ admitting polynomial inequalities. Proc. Steklov Inst. Math. $\mathbf{3}$, 361-366 (1995)

20. Wallin, H., Wingren, P.: Dimensions and geometry of sets defined by polynomial inequalities. J. Approx. Theory 69, 231-249 (1992)

21. Zeriahi, A.: Inégalité de Markov et développement en série de polynômes orthogonaux des fonctions $\mathcal{C}^{\infty}$ et $\mathcal{A}^{\infty}$. In: Fornaess, J.F. (ed.) Proceedings of the Special Year of Complex Analysis of the Mittag-Leffler Institute 1987-88, pp. 693-701. Princeton Univ. Press, Princeton New Jersey (1993) 